\title{
Adult-onset Still's disease and the role of dermatological manifestations: A case report and literature review
}

\author{
ANDREI VÂȚĂ $\breve{~}^{1,2}$, IONELA ADRIANA VLĂDUȚU ${ }^{3}$, CARMEN MANCIUC $^{1,2}$, \\ CRISTINA LĂCĂTUSU ${ }^{4}$, ALEXANDRA BURLUI $^{5}$, ANCA CARDONEANU $^{5}$ and ELENA REZUȘ ${ }^{5}$ \\ ${ }^{1}$ Department of Infectious Diseases, 'Grigore T. Popa' University of Medicine and Pharmacy, \\ 700115 Iași; ${ }^{2}$ Department of Infectious Disease, Clinical Hospital of Infectious Diseases, 700116 Iași; \\ ${ }^{3}$ Department of Internal Medicine, 'Dr. C.I. Parhon' Clinical Hospital, 700503 Iași; Departments of ${ }^{4}$ Diabetes and \\ Metabolic Disorders, and ${ }^{5}$ Rheumatology, 'Grigore T. Popa' University of Medicine and Pharmacy, 700115 Iași, Romania
}

Received May 29, 2020; Accepted June 30, 2020

DOI: $10.3892 /$ etm.2020.9515

\begin{abstract}
Adult-onset Still's disease (AOSD) is a rare inflammatory systemic disease with unknown etiology, characterized by spiking fever, evanescent rash, arthralgia and arthritis, leukocytosis and possible multi-organ involvement. Based on a case report of a 19-year-old man who was admitted to hospital for an influenza-like syndrome associated with a transient and recurrent pale-red disseminated non-specific rash, we performed a PubMed database search for cases and reviews of Adult's Onset Still's Disease published in English in the last 5 years. Due to its heterogeneous clinical manifestations, of which skin rash is an important sign, and the lack of a specific laboratory test, the diagnosis requires the exclusion of a wide range of mimicking disorders and the use of specific scoring systems. The high ferritin levels, major leukocytosis with neutrophilia, absence of typical antibodies for other systemic autoimmune diseases and other markers of infectious disease were the milestones that led to the positive diagnosis. The first line of treatment remains corticosteroid therapy in association with disease-modifying anti-rheumatic drugs.
\end{abstract}

\section{Introduction}

Adult-onset Still's disease (AOSD) is a rare inflammatory condition (annual incidence of 0.1-0.4 new cases per 100,000 inhabitants) associating articular and systemic symptoms, which may occur at any age, although in $75 \%$ of the cases the onset of the disease is between the ages of 16 and 35 , with a relatively equal distribution between the two sexes (1).

Correspondence to: Dr Carmen Manciuc, Department of Infectious Diseases, 'Grigore T. Popa' University of Medicine and Pharmacy, 16 Universitatii Street, 700115 Iasi, Romania

E-mail: c.manciucumf@gmail.com

Key words: rash, fever, ferritin, autoimmunity, inflammation

\section{Case report}

We report the case of a 19-year-old man without any personal medical history who was hospitalized at the peak of the flu epidemic season for fever as high as $39.5^{\circ} \mathrm{C}$, odynophagia, dysphagia, generalized myalgia, dry cough and impaired general condition. These symptoms had started 10 days earlier. The patient's general practitioner considered this condition to be a respiratory infection and prescribed Clarithromycin and Ibuprofen, but after 5 days of treatment, erythematous maculo-papular and slightly pruriginous exanthema occurred on the patient's trunk, upper limbs and face, and was managed as an allergic reaction. It disappeared immediately after antihistaminic drug administration, but the fever persisted and the myalgia worsened.

On hospitalization, the patient had a fever $\left(38.7^{\circ} \mathrm{C}\right)$, acne lesions on the posterior thorax, diffuse pharyngeal congestion, multiple mobile, painless, infracentimetric submandibular adenopathies on both sides. He was slightly tachycardic, and he exhibited elastic hepatomegaly, $3 \mathrm{~cm}$ below the costal margin. The presence of intense myalgia made it difficult for him to actively or passively move or walk.

Considering the clinical picture and the epidemic context, the diagnosis of influenza was suspected and Oseltamivir therapy was initiated just before the results of the first laboratory tests that showed: leukocytosis with neutrophilia, important inflammatory biological syndrome, discrete hepatocytolisis (Table I). The rapid test for influenza A and B virus antigens in nasopharyngeal exudate was negative; therefore, the antiviral therapy was discontinued.

Chest X-ray revealed bilateral interstitial infiltrates, and the abdominal ultrasound scan confirmed the presence of a homogeneous hepatomegaly and also reveals splenomegaly (140 mm bipolar diameter).

Antibiotic treatment with Ceftriaxone was initiated, and for the next 3 days, myalgia and cough improved, yet the patient still experiences daily fever episodes (especially at night) $\leq 39.2^{\circ} \mathrm{C}$. On the $3 \mathrm{rd}$ day, he developed again an erythematous maculo-papular pale exanthema, similar to the one the patient suffered before hospitalization. Desloratadine was administered and the antibiotic treatment was reformed 
Table I. Evolution of hematological and biochemical parameters.

\begin{tabular}{lrrrrrrr}
\hline Day (of hospitalization) & \multicolumn{1}{c}{1} & \multicolumn{1}{c}{5} & \multicolumn{1}{c}{11} & 13 & 15 & 28 & 42 \\
\hline White blood cells/mm & 18,270 & 15,260 & 36,030 & 12,510 & 24,620 & 22,400 & 11,200 \\
Neutrophils (\%) & 84 & 86 & 93 & 86 & 90 & 83 & 74 \\
ESR (mm/h) & 90 & 75 & 95 & 110 & 100 & 65 & 30 \\
Fibrinogen (g/l) & 6.4 & 4.2 & 7.6 & 7.1 & 7.2 & & \\
CRP & 160 & 140 & 208 & 110 & 95 & & \\
ALT (UI/l) & 46 & 71 & 105 & 100 & 105 & 45 & \\
AST (UI/l) & 65 & 81 & 59 & 56 & 51 & & \\
CK (U/l) & 36 & & 42 & & & & \\
LDH (U/l) & 402 & & 381 & & & &
\end{tabular}

ESR, erythrocyte sedimentation rate; CRP, C-reactive protein; ALT, alanine aminotransferase; AST, aspartate aminotransferase; CK, creatine kinase; LDH, lactate dehydrogenase.

using Moxifloxacine and Vancomycin starting on the 7th day, as a systemic staphylococcal infection was suspected. The patient remained feverish, and experienced abundant sweating and variably severe myalgia.

Hemocultures sampled during a fever episode on hospitalization day remained negative, just like urine culture and pharyngeal exudate. The HIV test was negative. Infectious endocarditis was suspected, but he had no heart murmur, or abnormal echocardiographic findings. The IgM antibodies against Mycoplasma pneumoniae were negative.

The clinical picture and the presence of hepatocytolysis raised suspicion of infectious mononucleosis, but anti-VCA IgM antibodies were absent. There was also an assumption of a possible Toxocara canis infection (fever, hepatomegaly, exanthema), but the patient did not exhibit eosinophilia or anti-parasite antibodies.

Starting on the 12 th day, he received Amikacin + Piperacillin/Tazobactam treatment, but the fever and leukocitosis persisted.

Another chest X-ray performed on the 14th day of hospitalization showed that the initial interstitial infiltrate had disappeared, but it also revealed the presence of right hilum adenopathy and the hypothesis of pulmonary tuberculosis or sarcoidosis was raised. Therefore, a fibro-bronchoscopic examination and broncho-alveolar lavage were performed. The microscopic examination after Zielh-Nielssen staining did not reveal Koch bacilli and later the culture was negative.

Among the non-infectious causes of prolonged fever syndrome associated with myalgia and recurrent exanthema, and intense inflammatory syndrome, we also listed systemic collagenosis, but the whole panel of nuclear anti-antigen (anti-nRNP/Sm, anti-Sm, anti-SS-A (Ro), anti-Ro-52, anti-SS-B (La), anti-Scl-70, anti-PM-Scl100, anti-Jo-1, antiCentromer B, anti-PCNA, anti-dsDNA, anti-Nucleozome, anti-histone, anti-ribosomal protein $\mathrm{P}$, anti-AMA-M2, antiDFS70) antibodies was negative. Quantification of muscle sufferance was attempted by determining serum creatine phosphokinase, the values of which were repeatedly normal, LDH values $\sim 2$ times the normal value and slightly higher serum aldolase values (12 U/1 ) (Table I). On the 14th day, we conducted musculocutaneous biopsy, which only revealed minor non-characteristic morphological changes that allowed the diagnosis of dermatomyositis to be ruled out; no sarcoidosis-like granulomatous inflammation was reported. Another hypothesis was the presence of a malignant hematological disease, but the hematologist did not support it due to the aspect of the blood smear (leukemoid reaction with the deviation of the leukocytic formula to the neutrophil myelocytic stage with the presence of toxic granulations and vacuolizations in the neutrophil leukocyte cytoplasm, lymphopenia).

Starting on the 16th day, the clinical picture was dominated by the presence of inflammatory arthralgia of initially oligoarticular and migratory type with the involvement of knee and shoulder joint. Subsequently, the evolution was polyarticular and fixed type with a tendency to symmetry with bilateral fist joint and small hand joint impairment (bilateral metacarpophalangeal joints I-V, bilateral interphalangeal joints I-V). At this point, the suspected diagnosis was AOSD. The tests showed very high serum ferritin levels - 7,354 ng/ml (N: 21.8-274.7) (the glycosylated fraction of ferritin could not be determined).

Together with the rheumatologist, since another infectious or non-infectious cause of his suffering could not be identified, we started the Solumedrol $125 \mathrm{mg} /$ day therapy, which led to the partial remittance of his clinical symptoms.

The current musculoskeletal system exam revealed pain on palpation of the topographic points of both shoulders and limited movement in any direction of both shoulders due to pain, pain on bilateral palpation of the radio-cubito-carpal joint space and interphalangeal joints $\mathrm{I}-\mathrm{V}$ without joint swelling, with preserved grip strength and fine precision, and painful knee joint on palpation of the cul-de-sac and cracking noise on both joint mobilization, otherwise normal from the osteoarticular point of view.

The biological tests indicate the persistence of an important inflammatory syndrome and no immunological changes (negative Latex and W. Rose rheumatoid factor, cANCA, pANCA, C3, C4 with normal values), thus ruling out a diagnosis of ANCA-related vasculitis.

Correlation of clinical and laboratory data confirmed the diagnosis of AOSD, as 7 of the 8 Yamagouchi diagnosis criteria were met (Table II), of which 4 major criteria (high 
Table II. Yamaguchi classification criteria (24).

Major criteria

Minor criteria

Intermittent fever of at least $39^{\circ} \mathrm{C}$, which lasts $\geq 1$ week

Arthralgia lasting $\geq 2$ weeks

Specific rash (Still rash)

Leukocytosis $\left(\geq 10,000 / \mathrm{mm}^{3}\right)$, with $\geq 80 \%$ granulocytes

Exclusion criteria

Infections

Malignant tumors

Other rheumatic conditions
Sore throat

Recent development of significant lymphadenopathy Hepatomegaly or splenomegaly

Abnormal liver function

Negative tests for antinuclear antibodies and rheumatoid factor

Diagnosis $=5$ criteria, of which at least 2 major.

Table III. Classification criteria developed by Fautrel et al (25).

Major criteria Minor criteria

High fever $>39^{\circ} \mathrm{C}$

Arthralgia

Transient erythematous rash

Sore throat

Polymorphonuclear cells $\geq 80 \%$

Glycosilated ferritin $\leq 20 \%$

Diagnosis $=4$ major criteria or 3 major criteria +2 minor .

fever over $39^{\circ} \mathrm{C}$ lasting over one week, arthralgia longer than 2 weeks, Still rash, leukocytosis $>10,000 / \mathrm{mmc}$ with granulocytosis over $80 \%$ ) and 3 minor criteria (splenomegaly, negative for rheumatoid factor and antinuclear antibodies, changes in serum liver enzyme concentration). The Fautrel diagnosis criteria (Table III) were also met, more precisely 5 of the major ones (high fever $>39^{\circ} \mathrm{C}$, arthralgia, specific skin rash and odynophagia).

Corticoid therapy was continued in association with Methylprednisolone $125 \mathrm{mg} / \mathrm{i} . \mathrm{v}$ pulse therapy, 1 pulse per day for 3 days, and then $1 \mathrm{mg} / \mathrm{kg} /$ day $(64 \mathrm{mg}$ ) and Medrol by oral administration. The fever slowly remitted and the skin lesions disappeared. Given the patient's slow recovery and partial persistence of joint symptoms, we found it appropriate to associate $15 \mathrm{mg}$ of Methotrexate per week to the corticotherapy.

The patient was discharged from hospital with no fever, and improved general health status due to the absence of specific clinical symptoms, and he was ordered to continue the Medrol therapy at home $(64 \mathrm{mg} /$ day, which should be progressively reduced to $8 \mathrm{mg} /$ week), adding Pantoprazole $40 \mathrm{mg} / \mathrm{day}$, Methotrexate $15 \mathrm{mg} /$ week and folic acid $5 \mathrm{mg} /$ week $48 \mathrm{~h}$ after Methotrexate administration.

We searched PubMed database for full text cases and reviews of AOSD published in English up to 5 years ago. The search terms were: Adult Still Disease. Authors independently scanned the full text articles for the following inclusion criteria: i) cases of Adult's Onset Still's Disease, ii) published in English language, and iii) literature review. We also scrutinized the reference lists of the included articles to identify additional references. The used search strategy identified 159 publications (22 reviews) of which 28 were included for further analysis.

All procedures performed were in accordance with the ethical standards of the institutional and/or national research committee and with the 1964 Declaration of Helsinki and its later amendments or comparable ethical standards. The study was approved by the Ethics Committee of the Clinical Hospital of Infectious Diseases (Iasi, Romania). Written informed consent was obtained from the patient prior to submission of this article for consideration as a case based review.

\section{Discussion}

Adult-onset Still's disease - possible triggers and pathogenic mechanisms. In 1897, Sir George Frederick Still first reported cases of chronic polyarthritis children experiencing fever and lymphadenopathy, this condition currently being included in the systemic subtype of juvenile idiopathic arthritis. In 1971, Eric Bywaters described adults with clinical symptoms similar to those reported by the pediatrician Still in children, which did not meet the rheumatoid arthritis criteria, thus defining a new clinical entity: the AOSD (2).

The clinical presentation of AOSD is polymorphic, with specific manifestations depending on the time of the patient's examination, with an important systemic and visceral component that influences the severity of the disease, the differential diagnosis requiring the exclusion of a wide range of conditions such as infectious diseases, lymphoproliferative diseases, vasculitis, sarcoidosis, autoinflammatory diseases, neutrophilic dermatoses and, last but not least, autoimmune rheumatic diseases such as rheumatoid arthritis, systemic lupus erythematosus, idiopathic inflammatory myopathies and reactive arthritis, which is why the accurate diagnosis of the disease is often delayed.

The AOSD etiology remains unclear, despite several hypotheses that have been formulated. Viral infections, genetic factors and immune dysfunction, cytokine-mediated inflammation and apoptosis disorder may be involved in the pathogenesis of this disease.

The involvement of a triggering infectious agent has been suspected, among the incriminated microorganisms being 
rubella virus, measles, Echovirus 7, Coxsackievirus B4, Cytomegalovirus, Epstein-Barr virus, Human herpes virus 6, Parainfluenza, Influenza A, Adenovirus, Hepatitis B and C, Parvovirus B19 (3-7), Mycoplasma pneumonia, Chlamydia pneumonia, Yersinia enterocolitica, Brucella abortus, Borrelia burgdorferi (7), but a cause-effect relation has not been proven yet.

The susceptibility to inflammatory autoimmune reactions in response to the action of infectious factors is probably due to the existence of a genetic context. Although many studies have attempted to establish a correlation between genetic factors (HLA alleles) and disease onset, the results are limited and inconclusive due to the small number of patients diagnosed with this disease and to their ethnic diversity.

Recent advances in the medical field have highlighted that a key role in AOSD pathology is played by the activation of neutrophils and macrophages due to proinflammatory cytokine release (possibly through IL-18), which has led to the development of new therapeutic targets. Elevated TNF- $\alpha$ levels were detected in both serum and tissues of AOSD patient compared with the healthy group, values that did not correlate with disease activity (8). On the other hand, the serum level of the soluble tumor necrosis factor-receptor 2 (STNF-R2) correlated with the CRP level, may be used in the future as a disease activity marker (9). Elevated levels of IL-1 were detected in the serum of patients with untreated active disease, and IL-1 was found to be a major mediator of the inflammatory cascade, which is why IL-1 is a target for therapy. IL-1b is also a marker that could be used in assessing disease activity status and in monitoring the treatment (5). Elevated IL-6 levels were found in the blood of AODS patients compared with healthy subjects, this being dependent on disease activity, fever occurrence, and CRP increase. Moreover, skin biopsies from the specific salmon pink maculae were also sampled, which also revealed an elevated level of IL-6 $(10,11)$. The persistence of high IL-8 values is associated with joint impairment due to the disease, and it could also be used as a predictive marker (11). IL-18 in patients with AODS is predominantly found in the synovial fluid and lymph nodes, and may be used as a marker of the severity of the disease and response to corticoid therapy, the IL-18 level being in good correlation with ferritin (12).

Clinical manifestations. AOSD usually manifests itself as a trio of symptoms which include high fever, specific rash and arthritis/arthralgia (13).

The fever usually exceeds $39^{\circ} \mathrm{C}$, generally lasts under $4 \mathrm{~h}$ and most commonly occurs during the day, peaking in late afternoon or early evening, with rapid temperature variation of $>4$ degrees over the span of several hours and spontaneous remission. Fever may be associated with serositis, sore throat, myalgia and arthralgia. The overall incidence of fever in five of the largest retrospective studies was $95.7 \%$ (13-15).

Typically, the exanthema is a transient salmon-pink maculo-papular rash extending predominantly on the proximal limb areas and the trunk, with the rare involvement of the face and distal limb areas, accompanied by fever. It disappears between the fever episodes. The rash may be mildly pruritic and mistaken for drug allergy. Its incidence ranges between 51 and $87 \%$, with a mean value of $72.7 \%$ (16). In three cases, a purpura vascular skin rash has also been described and an association with mixed cryoglobulinemia has been suggested in one of them (17).

Arthralgia and arthritis are present in most AOSD patients, with the incidence ranging between 64 and 100\% $(16,17)$. Most often, the affected joints are the shoulders, knees, fists and ankles, although the involvement of the cervical spine, proximal and, more rarely, distal interphalangeal joints, metacarpo-phalangeal and metatarso-phalangeal joints, temporo-mandibular joints and hip was also described. In particular, carpal and pericapitate abnormalities are usually more marked than in cases of rheumatoid polyarthritis, providing a means of clinically distinguishing between the two entities. It is noteworthy that the changes in the hand joint typically occur 6 months after the onset of the disease, with a progressive reduction of the joint space in a pericapitate or carpometacarpal distribution, and ankylosis that develops after $1.5-3$ years $(1,18-21)$. The arthritis model is typically symmetrical, with most patients having polyarticular impairment and pain associated with the fever spikes. These symptoms are often short-lived, and they remit as the fever diminishes.

Myalgia is another common manifestation, its incidence raging between 56 and $84 \%$ (16). Its distribution remains generalized and, most often, occurs with exacerbations of fever.

Hepatic abnormalities, predominantly hepatomegaly and hepatic biochemistry abnormalities, are common among $\sim 50-75 \%$ of patients, but it has been suggested that hepatic dysfunction due to the use of non-steroidal anti-inflammatory drugs (NSAIDs) may be a significant cofactor $(13,20)$. AOSD may have the appearance of pseudo-angiocolitis. Jaundice and acute hepatitis leading to acute liver failure requiring liver transplantation remain extremely rare (22).

Lymphadenopathy occurs in $33-73 \%$ of patients with palpable and sensitive cervical ganglions, non-adhering to the deep structures; immune-histochemical tests have been used to reveal the benign polyclonal hyperplasia of B lymphocytes.

Diagnostic algorithms. The AOSD diagnosis still remains a clinical one. Unlike other systemic rheumatic diseases, it is not associated with positive rheumatoid factor (RF) or antinuclear antibody (ANA). The laboratory profile of the disease is a reflection of systemic inflammation and of the presence of the cytokine cascade.

The definitive diagnosis of Still's disease is difficult and different authors proposed several lists of symptoms and laboratory parameters to help the clinician, all based on retrospective studies. A precise evaluation of these criteria is difficult due to the rarity of the disease and the lack of a control group. Considering the 1996 Masson et al (23) comparative study, the criteria proposed by Yamaguchi et al (24) were the most sensitive (Table II). Fautrel et al (25) criteria (Table III) took into account the level of the glycosylated fraction of serum ferritin and different exclusion criteria achieving better performances: $80.6 \%$ sensitivity and $98.5 \%$ specificity.

Serum ferritin and glycosylated ferritin have recently received great attention as markers of disease diagnosis and activity. Inflammation is associated with increased ferritin production through the histocytic-macrophage system and/or significant release of damaged hepatocytes. Ferritin levels in AOSD are usually higher than the ones specific to patients 
with other autoimmune or inflammatory diseases. In most studies, the threshold used for serum ferritin concentrations to suggest AOSD was $1,000 \mathrm{ng} / \mathrm{ml}$, i.e., five times the upper limit of normal values (40-200 ng/ml) (26). Very high levels, from $4,000 \mathrm{ng} / \mathrm{ml}$ to $30,000 \mathrm{ng} / \mathrm{ml}$, are not uncommon and even extreme levels of up to $25,0000 \mathrm{ng} / \mathrm{ml}$ have been recorded. Furthermore, levels of serum ferritin correlate with disease activity and often normalize when the disease remits (16). The validity of hyperferritinemia as a diagnostic tool was evaluated in a French retrospective study, where a five-fold increase in serum ferritin had $80 \%$ sensitivity and $41 \%$ specificity (27) and, similarly, in a Japanese study (24) sensitivity was $82 \%$ and specificity was $46 \%$.

Better diagnostic tests are needed, and new immunological tests, such as IL18, may prove useful in the near future for diagnosis setting, as well as for monitoring disease activity and response to treatment. In the end, the positive diagnosis is an exclusion diagnosis, and differential diagnosis may take a long time.

Therapeutic options and prognosis. The treatment of AOSD patients has been an empirical one due to the lack of concrete data from randomized double-blind trials, the small number of cases, and the findings of retrospective studies $(12,28)$. The first line of treatment remains corticosteroid therapy in association with disease-modifying anti-rheumatic drugs (DMARDS). The maintenance dose is $0.5-1 \mathrm{mg} / \mathrm{kg} / \mathrm{day}$ of prednisone equivalent (29). If there is no effective therapeutic control after 2 months of treatment with glucocorticoids, another therapeutic option is methotrexate, cyclosporine, hydroxychloroquine, or azathioprine. Methotrexate remains the most commonly used DMARD, in a treatment plan similar to the one used for rheumatoid arthritis, which occupies a central place in patients with corticosteroid addiction (13).

Conclusions. Although not very frequent, AOSD may pose a real challenge to the clinician. Progress has been made in understanding the immunological pathways of the disease, but further research is needed for the development of a reliable diagnostic tool and better treatment options.

The case reported here falls relatively well in the AOSD definition and characteristics, with clinical manifestations dominated by prolonged fever, refractory to the administered anti-infective therapy, associated with particularly important and debilitating myalgias, and generalized cyclical exanthema. One of the particularities of the presented case was the late occurrence of articular manifestations. The very high ferritin levels, major leukocytosis with neutrophilia, absence of typical antibodies for other systemic autoimmune diseases and other markers of infectious disease were the milestones that led to the patient's diagnosis, which a multidisciplinary team helped to set.

\section{Acknowledgements}

Not applicable.

\section{Funding}

No funding was received.

\section{Availability of data and materials}

All data generated or analyzed during this review are included in this published article.

\section{Authors' contributions}

AV, IAV, ER, CM, AB, AC and CL designed the study, analyzed and interpreted the patient data and drafted the manuscript. AV revised the manuscript and made substantial intellectual contributions to the study. All authors read and approved the final manuscript.

\section{Ethics approval and consent to participate}

All procedures performed in the present study involving human participants were in accordance with the ethical standards of the institutional and/or national research committee and with the 1964 Declaration of Helsinki and its later amendments or comparable ethical standards. The study was approved by the Ethics Committee of the Clinical Hospital of Infectious Diseases (Iasi, Romania).

\section{Patient consent for publication}

Written informed consent was obtained from the patient prior to submission of this article for consideration as a case-based review.

\section{Competing interests}

The authors declare that they have no competing interests.

\section{References}

1. Hochberg M, Gravallese E, Silman A, Smolen J, Weinblatt M and Weisman M (eds): Rheumatology. 7th edition. Elsevier, 2018.

2. Bywaters EG: Still's disease in the adult. Ann Rheum Dis 30: 121-133, 1971.

3. Ohta A, Yamaguchi M,Tsunematsu T, Kasukawa R, Mizushima H, Kashiwagi H, Kashiwazaki S, Tanimoto K, Matsumoto Y, Akizuki M, et al: Adult Still's disease: A multicenter survey of Japanese patients. J Rheumatol 17: 1058-1063, 1990.

4. Wouters JM, van der Veen J, van de Putte LB and de Rooij DJ: Adult onset Still's disease and viral infections. Ann Rheum Dis 47: 764-767, 1988.

5. Escudero FJ, Len O, Falcó V, de Sevilla TF and Sellas A: Rubella infection in adult onset Still's disease. Ann Rheum Dis 59: 493, 2000.

6. van de Putte LB and Wouters JM: Adult-onset Still's disease. Baillieres Clin Rheumatol 5: 263-275, 1991.

7. Gerfaud-Valentin M, Jamilloux Y, Iwaz J and Sève P: Adult-onset Still's disease. Autoimmun Rev 13: 708-722, 2014.

8. Hoshino T, Ohta A, Yang D, Kawamoto M, Kikuchi M, Inoue Y, Kamizono S, Ota T, Itoh K and Oizumi K: Elevated serum interleukin 6 , interferon-gamma, and tumor necrosis factor-alpha levels in patients with adult Still's disease. J Rheumatol 25: 396-398, 1998.

9. Fujii T, Nojima T, Yasuoka H, Satoh S, Nakamura K, Kuwana M, Suwa A, Hirakata M and Mimori T: Cytokine and immunogenetic profiles in Japanese patients with adult Still's disease. Association with chronic articular disease. Rheumatology (Oxford) 40: 1398-1404, 2001.

10. Choi JH, Suh CH, Lee YM, Suh YJ, Lee SK, Kim SS, Nahm DH and Park HS: Serum cytokine profiles in patients with adult onset Still's disease. J Rheumatol 30: 2422-2427, 2003.

11. Chen DY, Lan JL, Lin FJ and Hsieh TY: Proinflammatory cytokine profiles in sera and pathological tissues of patients with active untreated adult onset Still's disease. J Rheumatol 31: 2189-2198, 2004 
12. Mavragani CP, Spyridakis EG and Koutsilieris M: Adult-onset Still's disease: From pathophysiology to targeted therapies. Int J Inflam 10: 879020, 2012.

13. Wouters JM and van de Putte LB: Adult-onset Still's disease; clinical and laboratory features, treatment and progress of 45 cases. Q J Med 61: 1055-1065, 1986.

14. Pouchot J, Sampalis JS, Beaudet F, Carette S, Décary F, Salusinsky-Sternbach M, Hill RO, Gutkowski A, Harth M, Myhal D, et al: Adult Still's disease: Manifestations, disease course, and outcome in 62 patients. Medicine (Baltimore) 70 : 118-136, 1991.

15. Kádár J and Petrovicz E: Adult-onset Still's disease. Best Pract Res Clin Rheumatol 18: 663-676, 2004.

16. Efthimiou P, Paik PK and Bielory L: Diagnosis and management of adult onset Still's disease. Ann Rheum Dis 65: 564-572, 2006

17. Elezoglou AV, Giamarelos-Bourboulis E, Katsilambros N and Sfikakis PP: Cutaneous vasculitis associated with mixed cryoglobulinemia in adult Still's disease. Clin Exp Rheumatol 21: 405-406, 2003

18. Björkengren AG, Pathria MN, Sartoris DJ, Terkeltaub R, Esdaile JM, Weisman M and Resnick D: Carpal alterations in adult-onset Still disease, juvenile chronic arthritis, and adult-onset rheumatoid arthritis: Comparative study. Radiology 165: 545-548, 1987.

19. Medsger TA Jr and Christy WC: Carpal arthritis with ankylosis in late onset Still's disease. Arthritis Rheum 19: 232-242, 1976.

20. Grajdeanu IA, Statescu L, Vata D, Grigorescu C, Popescu I-A, Porumb Andrese E, Taranu T, Patrascu A and Solovastru Gheuca L: Contribution of imaging techniques in the management of cutaneous pathology. Rev Chim 70: 87-91, 2019.

21. Mare LV, Andrese EP, Monu AM, Adam MG, Solovastru LG and Vata D: Update on epidemiology of non-melanocytic skin tumors. Rev Chim 70: 3050-3052, 2019.
22. Andrès E, Kurtz JE, Perrin AE, Pflumio F, Ruellan A, Goichot B, Dufour P, Blicklé JF, Brogard JM and Schlienger JL: Retrospective monocentric study of 17 patients with adult Still's disease, with special focus on liver abnormalities. Hepatogastroenterology 50: 192-195, 2003.

23. Masson C, Le Loet X, Liote F, Dubost JJ, Boissier MC, Perroux-Goumy L, Bregeon C and Audran M: Comparative study of 6 types of criteria in adult Still's disease. J Rheumatol 23: 495-497, 1996.

24. Yamaguchi M, Ohta A, Tsunematsu T, Kasukawa R, Mizushima Y, Kashiwagi H, Kashiwazaki S, Tanimoto K, Matsumoto Y, Ota T, et al: Preliminary criteria for classification of adult Still's disease. J Rheumatol 19: 424-430, 1992.

25. Fautrel B, Zing E, Golmard JL, Le Moel G, Bissery A, Rioux C, Rozenberg S, Piette JC and Bourgeois P: Proposal for a new set of classification criteria for adult-onset still disease. Medicine (Baltimore) 81: 194-200, 2002.

26. Fautrel B: Ferritin levels in adult Still's disease: Any sugar? Joint Bone Spine 69: 355-357, 2002.

27. Fautrel B, Le Moël G, Saint-Marcoux B, Taupin P, Vignes S, Rozenberg S, Koeger AC, Meyer O, Guillevin L, Piette JC, et al: Diagnostic value of ferritin and glycosylated ferritin in adult onset Still's disease. J Rheumatol 28: 322-329, 2001.

28. Bagnari V, Colina M, Ciancio G, Govoni $\mathrm{M}$ and Trotta F: Adult-onset Still's disease. Rheumatol Int 30: 855-862, 2010.

29. Masson C, Le Loët X, Lioté F, Renou P, Dubost JJ, Boissier MC, Brithmer L, Brégeon C and Audran M: Adult Still's disease. Part II. Management, outcome, and prognostic factors. Rev Rhum Engl Ed 62: 758-765, 1995.

This work is licensed under a Creative Commons Attribution-NonCommercial-NoDerivatives 4.0 International (CC BY-NC-ND 4.0) License. 